\title{
On Construction of Intelligent Archives Management System in Colleges and Universities
}

\author{
Dan ZHANG ${ }^{1 *}$, Lie-sheng $\mathrm{HUANG}^{2}$, Zi-heng HUANG ${ }^{3}$ \\ ${ }^{1}$ Shandong Technology \& Business University \\ ${ }^{2}$ Shandong Technology \& Business University \\ ${ }^{3}$ Yantai Vocational College of Culture and Tourism \\ ${ }^{*}$ Corresponding author: Dan ZHANG,Email:danazhang2004@ 163.com
}

\begin{abstract}
The traditional archive management mode of colleges and universities cannot meet the needs of the construction and development of colleges and universities, Through the construction of intelligent archives management system of colleges and universities, intelligent means are used to change the archive management idea, change the management mode, innovate the service mode, perfect the relevant system, improve the level of management and the security ability, improve the efficiency and service ability of archives work. It is helpful to provide safe, accurate, personalized and efficient intelligent services for teachers and students in colleges and universities.
\end{abstract}

Keywords: Intelligent archives, System, Function

\section{INTRODUCTION}

University archives work is not only an important part of the construction and development in colleges and universities, but also one of the important resources in colleges and universities. University archives cover a wide range of areas and have rich archive resources, which provide accurate decision-making data for university planning and development. With the increase of the university construction time, the variety and quantity of archives grow rapidly. Archives work plays a great role in teaching and scientific research services. Traditional archives management and simple face-to -face inquiry restrict the improvement of archives work efficiency. It is difficult to adapt to the continuous utilization requirements from the inside and outside of universities. The rapid development of information construction promotes the construction process of the intelligent archives ${ }^{[1]}$. It is urgent to use modern, digital and intelligent technologies to establish a perfect system of archives resources, convenient file use archives system and security system for teachers and students, so that file management idea can be changed and archives management model can be innovated. Consequently, it is helpful to raise the level of the university archives management, to enhance archival security ability, to improve archival work efficiency, to enhance archival service ability and realize safe, accurate, fast and efficient management mode. It is better to provide intelligent services for teachers and students in colleges and universities ${ }^{[2]}$.

\section{ADVANTAGES \& CHARACTERISTICS OF INTELLIGENNT ARCHIVES MANAGEMENT SYSTEM}

Intelligent archives management system is a collection of content management system, integration system and digital information long-term storage system. It mainly manages unstructured data such as electronic files, archives and other information resources. It is not only a data center but also a release and utilization center, and has the functions of orderly processing and integrated management. Intelligent archives management is beneficial to optimize and enhance the authenticity, integrity, effectiveness and order of archival information resources, and ultimately provide user demand-oriented archival information integration services.

The intelligent archives will subvert the traditional management mode of "focusing on storage and light utilization", mainly reflected in the characteristics of wide coverage, safety and reliability, convenience and quickness, and unified standards, rich resources and high degree of sharing ${ }^{[3]}$.Advantages of intelligent archives management system: 


\subsection{Innovative service mode ensures service quality and gives full play to the value of archives}

The utilization mode of "offline utilization" of traditional archives business is changed to the mode of "hierarchical and multi-channel archival information resource utilization". Different and diversified utilization modes are provided through online utilization, visiting library, integrated access, and information sharing. The traditional service mode of "passive consultation" will be changed into "active push". Service scopes are transformed from "archive professionals" to "full service". By providing multiple entries, the service channel of a "single channel" will be changed into "multiple interactions". Through the provision of full text retrieval, consultation, integrated portal, the development and utilization of archives service system, online interactive, online utilization of archives resources can be realized and the convenience of archives can be improved.

\subsection{Transforming data into digital resources, providing sustainable development capabilities.}

With the development of society, the thinking of archives is also changing ${ }^{[4]}$. Many archives have changed from the concept of "Paying attention to storage, neglecting to use" to "Paying attention to storage, neglecting to more use". The number of digital archives is increasing year by year, but most of them are not reasonably used and are only stored in the storage equipment of archives. The intelligent archives management system will turn these data resources into digital resources that can be more easily used, so that the ability of long-term sustainable development of digital resources in archives $^{[5]}$ can be enhanced. At the same time, archives resource management database can be built for receiving data, managing data, long-term storage of data, use of data storage and management. It ensures the rational allocation and rational use of archives resources

\subsection{Applying new technologies and means to improve the space used by archives}

With the rapid development of big data, intelligence and related technologies, technological innovation has promoted the progress of intelligence. The development of information technology will bring new opportunities and far-reaching influences to the development of archives, and new technologies and means can improve the utilization space of archives ${ }^{[6]}$.

\section{THE CONSTRUCTION OF INTELLIGENT ARCHIVES MANAGEMENT SYSTEM IN COLLEGES AND UNIVERSITIES}

The construction of intelligent archives management system covers standardized management of information resources, such as the collection, reception, sorting, storage, identification and statistics. It can improve the efficiency of business management and the security and reliability of resource services, and can meet the needs of users and archives managers.

\subsection{Security assurance subsystem}

\subsubsection{Separation function of "three kinds of staff"}

The separation design of system administrator, security administrator and security auditor is adopted, the responsibilities and division of labor of three kinds of staff is specified, and a sound mutual supervision mechanism is established. It can ensure the security and stability of the daily operation of the system, and the operation of the audit tracking analysis and supervision and inspection. It can timely detect the violations and regularly report to the relevant management authorities.

\subsubsection{Data encryption}

The encryption technology is used to encrypt data transmission and storage, ensuring end-to-end data transmission security, data transmission security from the browser to the application server, and the security of documents with different encryption levels.

\subsubsection{Permission Control Function}

Comprehensive permission management and control measures are taken to set up permissions for browsing, accessing, modifying, deleting, archiving, typing, downloading, printing, and management. It can help to finish permission management, such as display, search and preview, print, download, modification, publish, view logs, update versions and attachments management. When users need to download files, the archive administrator grants the download permission according to the actual situation. After users download files, the system can control offline data security.

\subsubsection{Identity Authentication function}

An independent identity authentication system is provided, which can control system login by user password. At the same time, the system also supports the combination with $\mathrm{CA}$ to achieve strong identity authentication. 


\subsubsection{Detection function of four characteristics}

By testing authenticity, integrity, availability and security of archive electronic files and metadata, automation and standardization can be realized, the disadvantage of inaccurate, non-standard, low efficiency and the drawbacks easily missed in artificial detection can be avoided, to ensure that electronic files will not be tampered with, process data will not be lost or omitted in the process of transfer, and the data is complete and compliant.

\subsubsection{Backup Restoration function}

The safety and backup management system is established, automatic backup in data management is achieved. The safety management of daily data will be combined with the construction of disaster recovery system.

\subsection{Collection archives management subsystem}

Collection archives management system is the most core business system of building intelligent archives and the foundation and resource of other related systems. The collection archives management system is mainly used to record, keep and utilize the collection data of the archives. It is a LAN platform for internal application of archives. To establish the archive core resources general database, the management of archives including various categories and various carriers can be realized.

\subsubsection{Archive collection and receiving function}

It mainly includes online collection, offline collection, transferring and receiving functions, it also includes the process of turning a completed file into a file after it has been transferred to storage in a receiving system, the archived files can be mounted and unloaded, identification and use, the functions are received by the resource administrator.

\subsubsection{Input management function}

It mainly includes the functions of archival description, scanning input, and transfer receiving information query. The direct input and collection of paper archives digitalization can be realized, and the function of viewing the details of the files handed over and received can be completed.

\subsubsection{Archives Management function}

It mainly includes the functions of file arrangement, file storage, file identification, and file statistics. The collected files are classified, arranged, numbered, catalogued and packed in units of pieces (volumes). It can maintain the archived information and manage the archived data, the data can be modified and exported, and the corresponding electronic files can be added to the whole volume information, it also has the functions of annual report verification, annual report summary and export.

\subsection{Electronic reading room subsystem}

The subsystem of electronic reading room is a multi-functional and modern reading place where integrates the retrieval, reading and service of digital archives, electronic documents and printed documents, and provides multimedia information service for users. The subsystem of electronic reading room includes the functions such as data management, utilization registration, utilization service, report statistics, release to portal, shortcut, recycle station, system management, and personal center. It imports the open data into the electronic reading room system for data utilization.

\subsubsection{Data Management Function}

It mainly includes import function, data collation, archiving data management, etc., to examine the details of collation files. It can make the users define complex retrieval conditions, and realize the accurate query of archives entries.

\subsubsection{Utilization and registration function}

After the user registration, the users' information registered in the system can be queried, including the user type, registration number, registration date, name, ID number, activation status and other information, and temporary accounts can be assigned to the visitors, it also includes modification and renewal of the registered staff and registration of the unregistered staff. The daily registration record function is used to record the registration of archivists on the same day.

\subsubsection{Utilization service functions}

The retrieval of archival digital resources handed over by the unit includes the retrieval methods of navigation retrieval, fuzzy retrieval, advanced combination retrieval, cross-database and cross-class retrieval, association retrieval, full-text inspection, the retrieval results can be sorted, exported, printed, and retrievably operated in the results, so that it can realize the reading function of many kinds of file formats, such as text files, online reading and readers.

\subsubsection{Statistical Reports}

There are two ways to provide customized statistical reports and user-defined statistical reports. The system provides the function of customized statistical reports, which can make statistics of collection, borrowing and utilization, project information, warehouse capacity and 
system data. Users can use user-defined statistical reports to customize different statistical reports based on different services.

\subsubsection{Personal Center}

It includes the functions of personal information, my collection, my subscription, download tools, messages, check file guidance, check file and guidance.

\subsection{Intelligent archives warehouse management subsystem}

Intelligent archives warehouse management subsystem will transform management ways from a single, independent, scattered traditional warehouse management method to a complex, comprehensive and linked modern warehouse management mode.

\subsubsection{High temperature-proof and moisture-proof subsystem}

The temperature and humidity automatic measurement and control management system is mainly composed of management host computer, intelligent module, temperature and humidity sensor, air conditioner, dehumidifier, humidifier and other devices.

3.4.2.Intelligent temperature and humidity measurement and control system of archive warehouse

It collects real- time temperature and humidity in monitoring area. It also supports linkage control with other subsystems.

\subsubsection{Air quality measurement and control system of archives}

It can remote monitor the status of the new fan, to ensure relatively good air quality in the warehouse.

\subsubsection{Water leakage monitoring system of archive warehouse}

Alarm is set for leakage module parameter, alarm event will be recorded and stored, and corresponding processing tips will be shown. It immediately sends out external alarm, such as multimedia voice, telephone/SMS, sound and light and wechat.

\subsubsection{Infrared anti-theft monitoring system of archive warehouse}

It monitors the alarm situation of each defense area in real time and realizes remote deployment and withdrawal through monitoring platform software.

\subsubsection{Dynamic security monitoring system of Archive warehouse}

In the monitoring center, the staff can monitor the activities of the main entrances and exits and the personnel of the passage through the image monitoring system. If abnormal conditions are found, the relevant personnel can be informed in time.

\subsubsection{Access control security monitoring system of archive warehouse}

The access control system in archive warehouse generally includes control devices, such as electronic lock, access control controller, fingerprint integrated machine and exit button, which can control the passing personnel and passing time in real time. It also can set the program control, and provide detailed records of all operations.

\section{CONCLUSIONS}

The establishment of the intelligent archives management system in colleges and universities greatly simplifies the working procedure. It implements remote monitoring and advance guidance for the electronic documents formed by various filing units. It avoids worthless files and duplicate files entering the library. It supports CD, floppy disk, network and other online/offline reception modes. It strengthens the protection of precious and important archives and improves the efficiency and level of archives utilization. It realizes the integrated management of files and archives, avoids the omission of files and materials, and improves the integrity and accuracy of files. It greatly improves the efficiency of archives management. It provides teachers and students with a more optimized storage environment and resource utilization environment. The construction of intelligent archives management system is of great significance, which can promote the digital archives to better provide the public with more intelligent services.

\section{REFERENCES}

[1] Peng Chen. Review on the Research of Domestic Intelligent Archives [J]. Lantai World, 2020 (12) pp:36-38.

[2] Li Yan. On Archives Informationization Construction in Higher Vocational Colleges Based on Intelligent Campus Environment [J]. Think Tank Times, 2019(42) pp:63.

[3] Kong Yang. Comparative Study on the Role of Intelligent Archives and Digital Archives in the Development of Archives. Archives Management 2020(5),Total Issue 246, pp:30 
[4] Zhao Yue, Wang Jun-hui. From digitization to Datamation: The Transformation of Archival Management Thinking. Archive Construction.

[5] He Long-long. Several Thoughts on the Construction of Intelligent Archives in the New Era. Think Tank Era. May 2019, pp:5

[6] Zhang li. Research on Information Construction of University Archives under the background of Intelligent Campus. August 2021, pp:15 